\title{
AUSLANDER-REITEN TRIANGLES IN DERIVED CATEGORIES OF FINITE-DIMENSIONAL ALGEBRAS
}

\author{
DIETER HAPPEL
}

(Communicated by Maurice Auslander)

\begin{abstract}
Let $A$ be a finite-dimensional algebra. The category $\bmod A$ of finitely generated left $A$-modules canonically embeds into the derived category $D^{b}(A)$ of bounded complexes over $\bmod A$ and the stable category $\bmod ^{\mathbb{Z}} T(A)$ of $\mathbb{Z}$-graded modules over the trivial extension algebra of $A$ by the minimal injective cogenerator. This embedding can be extended to a full and faithful functor from $D^{b}(A)$ to $\underline{\bmod }^{\mathbb{Z}} T(A)$. Using the concept of Auslander-Reiten triangles it is shown that both categories are equivalent only if $A$ has finite global dimension.
\end{abstract}

Let $k$ be a field and $A$ a finite-dimensional $k$-algebra. By $\bmod A$ we denote the category of finitely generated left $A$-modules. Let $T(A)$ be the trivial extension algebra by the bimodule $\operatorname{Hom}_{k}(A, k)$. Then $T(A)$ is a $\mathbb{Z}$-graded algebra and the category $\bmod ^{\mathbb{Z}} T(A)$ of finitely generated $\mathbb{Z}$-graded $T(A)$-modules is a Frobenius category in the sense of [H1]. The stable category of $\bmod ^{\mathbb{Z}} T(A)$ is denoted by $\bmod ^{\mathbb{Z}} T(A)$. In [H1] (see also [H2, KV]) we show that the derived category $D^{b}(A)=D^{b}(\bmod A)$ and $\bmod ^{\mathbb{Z}} T(A)$ are triangle-equivalent, if the global dimension of $A$ is finite. The purpose of this note is to prove the converse of this result. We point out that examples of this fact have been obtained in [TW]. Moreover we will give a surprisingly simple construction of a full and faithful exact functor $F$ from $D^{b}(A)$ to $\bmod ^{\mathbb{Z}} T(A)$.

The proof is based on the fact that $\bmod ^{\mathbb{Z}} T(A)$ has Auslander-Reiten triangles in the sense of $[\mathrm{H} 1]$, whereas we will show in section one that $D^{b}(A)$ has Auslander-Reiten triangles only if $A$ is of finite global dimension. Thus obtaining the converse of a result established in [H1].

In $\S 2$ we will give the construction of $F$ and the proof of the aforementioned theorem.

The composition of two morphisms $f: X \rightarrow Y$ and $g: Y \rightarrow Z$ in a given category is denoted by $f g$.

Received by the editors March 23, 1990.

1980 Mathematics Subject Classification (1985 Revision). Primary 16A46, 16A64, 18 E30.

Key words and phrases. Repetitive algebras, Auslander-Reiten triangles. 


\section{Auslander-Reiten triangles in $D^{b}(A)$}

In [H1] we introduced the notion of an Auslander-Reiten triangle in a triangulated category. We first recall the relevant definitions.

1.1. Let $\mathscr{C}$ be a triangulated category such that $\operatorname{Hom}_{\mathscr{C}}(X, Y)$ is a finitedimensional $k$-vector space for all $X, Y \in \mathscr{C}$ and assume that the endomorphism ring of an indecomposable object is local. This assumption ensures that $\mathscr{C}$ is a Krull-Schmidt category (compare 2.2 of [R]). We denote by $X[1]$ the value of the translation functor on the object $X$ of $\mathscr{C}$.

A triangle $X \stackrel{u}{\longrightarrow} Y \stackrel{v}{\longrightarrow} Z \stackrel{w}{\longrightarrow} X[1]$ in $\mathscr{C}$ is called an Auslander-Reiten triangle if the following conditions are satisfied:

(AR1) $X, Z$ are indecomposable;

(AR2) $w \neq 0$;

(AR3) if $f: W \rightarrow Z$ is not a retraction, then there exists $f^{\prime}: W \rightarrow Y$ such that $f^{\prime} v=f$.

We will say that $\mathscr{C}$ has Auslander-Reiten triangles if for all indecomposable objects $Z \in \mathscr{C}$ there exists a triangle satisfying the conditions above.

1.2. The following observations were obtained in [H1]. For more details and related results we refer to [H1, $\mathrm{H} 2]$.

First note that the following are equivalent for a triangle as above:

(i) (AR2);

(ii) $u$ is not a section;

(iii) $v$ is not a retraction.

If this is not the case we infer that $Y \simeq X \oplus Z$. Indeed, let $w=0$. Then we obtain the following diagram of triangles in $\mathscr{C}$

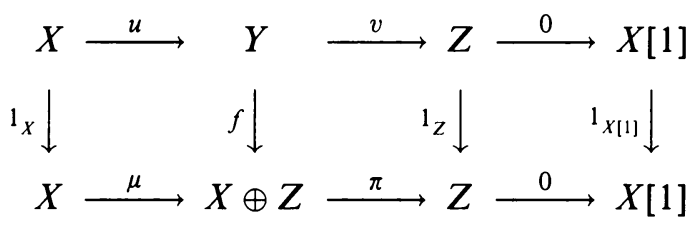

where $\mu$ and $\pi$ denote the canonical maps. Note that the second row is indeed a triangle, since it follows from the octahedral axiom that the direct sum of triangles is a triangle. Now we obtain by the third axiom of a triangulated category (see for example [V]) a morphism $f: Y \rightarrow X \oplus Z$, which is an isomorphism, by a well-known fact in triangulated categories (see for example [V]).

And also the following are equivalent for a triangle as above:

(i) (AR3);

(ii) If $f: W \rightarrow Z$ is not a retraction, then $f w=0$.

1.3. In the proof of the following theorem we will need some facts about $D^{b}(A)$ as well as the following notation. 
Let $\mathfrak{a}$ be an arbitrary additive subcategory of $\bmod A$. A complex $X^{\bullet}=$ $\left(X^{i}, d_{X}^{i}\right)_{i \in \mathbb{Z}}$ over $\mathfrak{a}$ is a collection of objects $X^{i}$ and morphisms $d^{i}=d_{X}^{i}$ : $X^{i} \rightarrow X^{i+1}$ such that $d^{i} d^{i+1}=0$. A complex $X^{\bullet}=\left(X^{i}, d_{X}^{i}\right)$ is bounded below if $X^{i}=0$ for all but finitely many $i<0$. It is called bounded above if $X^{i}=0$ for all but finitely many $i>0$. It is bounded if it is bounded below and bounded above. It is said to have bounded cohomology if $H^{i}\left(X^{\bullet}\right)=0$ for all but finitely many $i \in \mathbb{Z}$, where by definition $H^{i}\left(X^{\bullet}\right)=\operatorname{ker} d_{X}^{i} / \operatorname{im} d_{X}^{i-1}$. Denote by $C(\mathfrak{a})$ the category of complexes over $\mathfrak{a}$, by $C^{-, b}(\mathfrak{a})\left(\right.$ resp. $C^{+, b}(\mathfrak{a})$, resp. $\left.C^{b}(\mathfrak{a})\right)$ the full subcategories of complexes bounded above with bounded cohomology (resp. bounded below with bounded cohomology, resp. bounded above and below).

If $X^{\bullet}=\left(X^{i}, d_{X}^{i}\right)_{i \in \mathbb{Z}}$ and $Y^{\bullet}=\left(Y^{i}, d_{Y}^{i}\right)_{i \in \mathbb{Z}}$ are two complexes, a morphism $f^{\bullet}: X^{\bullet} \rightarrow Y^{\bullet}$ is a sequence of morphisms $f^{i}: X^{i} \rightarrow Y^{i}$ of a such that

$$
d_{X}^{i} f^{i+1}=f^{i} d_{Y}^{i}
$$

for all $i \in \mathbb{Z}$.

The translation functor is defined by $\left(X^{\bullet}[1]\right)^{i}=X^{i+1},\left(d_{X[1]}\right)^{i}=-\left(d_{X}\right)^{i+1}$.

The mapping cone $C_{f^{\bullet}}$ of a morphism $f^{\bullet}: X^{\bullet} \rightarrow Y^{\bullet}$ is the complex

$$
C_{f^{\bullet}}=\left(\left(X^{\bullet}[1]\right)^{i} \oplus Y^{i}, d_{C_{f}}^{i}\right)
$$

with 'differential'

$$
d_{C_{f}}^{i}=\left(\begin{array}{cc}
-d_{X}^{i+1} & f^{i+1} \\
0 & d_{Y}^{i}
\end{array}\right)
$$

We denote by $K^{-, b}(\mathfrak{a}), K^{+, b}(\mathfrak{a})$, and $K^{b}(\mathfrak{a})$ the homotopy categories of the categories of complexes introduced above.

Recall that two morphisms $f^{\bullet}, g^{\bullet}: X^{\bullet} \rightarrow Y^{\bullet}$ are called homotopic if there exist morphisms $h^{i}: X^{i} \rightarrow Y^{i-1}$ such that $f^{i}-g^{i}=d_{X}^{i} h^{i+1}+h^{i} d_{Y}^{i-1}$ for all $i \in \mathbb{Z}$.

For instance if $Z^{\bullet} \in C(\mathfrak{a})$ satisfies $Z^{i}=0$ for $i>0$, and $Z_{n}^{\bullet}$ for $n<0$ is the truncated complex with $Z_{n}^{i}=0$ for $i<n, Z_{n}^{i}=Z^{i}$ for $i \geq n, d_{Z_{n}}^{i}=d_{Z}^{i}$ for $i \geq n$, and zero otherwise, we obtain a morphism $\mu_{n}^{\bullet}: Z_{n}^{\bullet} \rightarrow Z^{\bullet}$ with $\mu_{n}^{i}=i d_{Z^{i}}$ for $i \geq n$ and zero otherwise whose mapping cone is isomorphic in the homotopy category to $Z_{n}^{\prime \bullet}$ with $Z_{n}^{\prime i}=0$ for $i \geq n$ and $Z_{n}^{\prime i}=Z^{i}$ for $i<n$ and $d_{Z_{n}^{\prime}}^{i}=d_{Z}^{i}$ for $i<n-1$ and zero otherwise. We denote by $\pi_{n}^{\bullet}$ the induced morphism from $Z^{\bullet}$ to $Z_{n}^{\prime \bullet}$. Thus $\pi_{n}^{i}=i d_{Z_{n}}$ for $i<n$ and zero otherwise. Note that $d^{n-1}$ induces a morphism $d_{n}^{\bullet}$ from $Z_{n}^{\prime \bullet}$ to $Z_{n}^{\bullet}[1]$. In particular we obtain a triangle in the homotopy category

$$
Z_{n}^{\bullet} \stackrel{\mu_{n}^{\bullet}}{\longrightarrow} Z^{\bullet} \stackrel{\pi_{n}^{\bullet}}{\longrightarrow} Z_{n}^{\prime \bullet} \stackrel{d_{n}^{\bullet}}{\longrightarrow} Z_{n}^{\bullet}[1]
$$

This construction will turn out to be quite useful in the next subsection. 
We denote by ${ }_{A} \mathscr{P}$ (resp. ${ }_{A}^{\mathscr{I}}$ ) the full subcategory of $\bmod A$ formed by the projective (resp. injective) $A$-modules. Then we identify the derived category $D^{b}(A)$ of bounded complexes over $\bmod A$ with $K^{-, b}\left({ }_{A} \mathscr{P}\right)$ or with $K^{+, b}\left({ }_{A} \mathscr{I}\right)$. In case $A$ has finite global dimension this yields the identification of $D^{b}(A)$ with $K^{b}\left({ }_{A} \mathscr{P}\right)$ or with $K^{b}\left({ }_{A} \mathscr{I}\right)$, since the natural embedding of $K^{b}\left({ }_{A} \mathscr{P}\right)$ into $K^{-, b}\left({ }_{A} \mathscr{P}\right)$ is an equivalence in this case.

1.4. In [H1] we proved that $D^{b}(A)$ has Auslander-Reiten triangles if the global dimension of $A$ is finite. Here we present now the following generaliztion.

Theorem. Let $A$ be a finite-dimensional k-algebra. Then

(i) Let $Z^{\bullet} \in K^{-, b}\left({ }_{A} \mathscr{P}\right)$ be indecomposable. Then there exists an Auslander-Reiten triangle $X^{\bullet} \rightarrow Y^{\bullet} \rightarrow Z^{\bullet} \rightarrow X^{\bullet}$ [1] if and only if $Z^{\bullet} \in K^{b}\left({ }_{A} \mathscr{P}\right)$.

(ii) Let $X^{\bullet} \in K^{+, b}\left({ }_{A} \mathscr{I}\right)$ be indecomposable. Then there exists an Auslander-Reiten triangle $X^{\bullet} \rightarrow Y^{\bullet} \rightarrow Z^{\bullet} \rightarrow X^{\bullet}[1]$ if and only if $X^{\bullet} \in K^{b}\left({ }_{A} \mathscr{I}\right)$.

Proof. We will prove (i). The second assertion follows by duality.

If $Z^{\bullet} \in K^{b}\left({ }_{A} \mathscr{P}\right)$ is indecomposable the construction of the Auslander-Reiten triangle

$$
X^{\bullet} \rightarrow Y^{\bullet} \rightarrow Z^{\bullet} \rightarrow X^{\bullet}[1]
$$

is identical as in the proof of 3.6 of [H1]. For the convenience of the reader we sketch the argument.

First observe that ${ }_{A} \mathscr{P}$ and ${ }_{A} \mathscr{I}$ are equivalent under the Nakayama functor $\nu=D \operatorname{Hom}_{A}\left(-,{ }_{A} A\right)$, where $D$ denotes the duality on $\bmod A$ with respect to the base field $k$. There is also an invertible natural transformation $\alpha_{P}: D \operatorname{Hom}(P,-) \rightarrow \operatorname{Hom}(-, \nu P)$ for $P \in \mathscr{P}$.

This induces an equivalence of triangulated categories again denoted by $\nu$ between $K^{b}\left({ }_{A} \mathscr{P}\right)$ and $K^{b}\left({ }_{A} \mathscr{I}\right)$ and an invertible natural transformation $\alpha_{P^{\bullet}}: D \operatorname{Hom}\left(P^{\bullet},-\right) \rightarrow \operatorname{Hom}\left(-, \nu P^{\bullet}\right)$ for $P^{\bullet} \in K^{b}\left({ }_{A} \mathscr{P}\right)$.

Now assume $Z^{\bullet}$ is indecomposable in $K^{b}\left({ }_{A} \mathscr{P}\right)$. Let $\varphi$ in $D \operatorname{Hom}\left(Z^{\bullet}, Z^{\bullet}\right)$ be a linear form on End $Z^{\bullet}$ which vanishes on the radical $\operatorname{rad} \operatorname{End} Z^{\bullet}$ and satisfies $\varphi\left(i d_{Z^{\bullet}}\right)=1$. We consider the image $\alpha_{Z^{\bullet}}(\varphi)$; it is a nonzero linear map from $Z^{\bullet}$ to $\nu Z^{\bullet}$ such that $f \alpha_{Z^{\bullet}}(\varphi)=0$ whenever the morphism $f$ of $D^{b}(A)$ is not a retraction. Consider the triangle

$$
\nu Z^{\bullet}[-1] \rightarrow Y^{\bullet} \rightarrow Z^{\bullet} \stackrel{\alpha_{Z^{\bullet}(\varphi)}}{\longrightarrow} \nu Z^{\bullet}
$$

having $\alpha_{Z^{\bullet}}(\varphi)$ as last morphism. We infer that this is an Auslander-Reiten triangle.

We are thankful to the referee for pointing out that the converse direction follows by applying proposition 3.2 of [Ril]. For the convenience of the reader we have included the elementary direct proof. 
For the converse let $Z^{\bullet} \in K^{-, b}\left({ }_{A} \mathscr{P}\right)$ be indecomposable. Applying the translation functor if necessary, we may assume that $Z^{i}=0$ for $i>0$. Assume that $Z^{\bullet}$ is not isomorphic to some complex in $K^{b}\left({ }_{A} \mathscr{P}\right)$ and let

$$
X^{\bullet} \stackrel{u^{\bullet}}{\longrightarrow} Y^{\bullet} \stackrel{v^{\bullet}}{\longrightarrow} Z^{\bullet} \stackrel{w^{\bullet}}{\longrightarrow} X^{\bullet}[1]
$$

be the Auslander-Reiten triangle. Set $W^{\bullet}=X^{\bullet}[1]$.

Let $n<0$ and consider the morphism $\mu_{n}^{\bullet}: Z_{n}^{\bullet} \rightarrow Z^{\bullet}$. We claim that $\mu_{n}^{\bullet}$ is not a retraction for all $n<0$. Assume that $\mu_{n}^{\circ}$ is a retraction. Then $Z_{n}^{\bullet} \simeq$ $Z_{n}^{\prime \bullet}[-1] \oplus Z^{\bullet}$ by the remark in 1.2. Since $Z^{\bullet}$ is not isomorphic to a complex in $K^{b}\left({ }_{A} \mathscr{P}\right)$ we obtain a contradiction.

From 1.2 it follows that $\mu_{n}^{\bullet} w^{\bullet}=0$ in $K^{-, b}\left({ }_{A} \mathscr{P}\right)$, (i.e. homotopic to zero) for all $n<0$. Let $h^{i}: Z_{n}^{i} \rightarrow W^{i-1}$ such that $\mu_{n}^{i} w^{i}=d_{Z_{n}} h^{i+1}+h^{i} d_{W}^{i-1}$ for all $i \in \mathbb{Z}$. Since $W^{\bullet} \in K^{-, b}\left({ }_{A} \mathscr{P}\right)$ there is $m_{0}<0$ such that $H^{m}\left(W^{\bullet}\right)=0$ for all $m \leq m_{0}$.

Let $n \leq m^{0}$.

We now define inductively $g^{i}: Z^{i} \rightarrow W^{i-1}$ such that $w^{i}=d_{Z}^{i} g^{i+1}+g^{i} d_{W}^{i-1}$ in contradiction to (AR2).

For $i>m_{0}$ we let $g^{i}=h^{i}$. By the considerations above and the fact that $\mu_{n}^{i}=i d_{Z^{i}}$ for $i \geq n$ we infer that

$$
w^{i}=d_{Z} g^{i+1}+g^{i} d_{W}^{i-1} \text { for } i>m_{0} .
$$

Now suppose that $g^{i}: Z^{i} \rightarrow W^{i-1}$ is defined such that $w^{i}=d_{Z} g^{i+1}+g^{i} d_{W}^{i-1}$ for $i>r \geq m_{0}$. Let $\pi^{r-1} \mu^{r-1}=d_{W}^{r-1}$ be the canonical factorisation of $d_{W}^{r-1}$. Consider $w^{r}-d_{Z}^{r} g^{r+1}: Z^{r} \rightarrow W^{r}$. Then

$$
\begin{aligned}
\left(w^{r}-d_{Z}^{r} g^{r+1}\right) d_{W}^{r} & =w^{r} d_{W}^{r}-d_{Z}^{r} g^{r+1} d_{W}^{r} \\
& =w^{r} d_{W}^{r}-d_{Z}^{r}\left(w^{r+1}-d_{Z}^{r+1} g^{r+2}\right) \\
& =w^{r} d_{W}^{r}-d_{Z}^{r} w^{r+1} \\
& =0 .
\end{aligned}
$$

Thus there exists $g^{\prime}: Z^{r} \rightarrow \operatorname{ker} d_{W}^{r}$ such that $g^{\prime} \mu^{r-1}=w^{r}-d_{Z}^{r} g^{r+1}$. Since $Z^{r}$ is a projective $A$-module there exists $g^{r}: Z^{r} \rightarrow W^{r-1}$ such that $g^{r} \pi^{r-1}=g^{\prime}$. Now

$$
\begin{aligned}
d_{Z}^{r} g^{r+1}+g^{r} d_{W}^{r-1} & =d_{Z}^{r} g^{r+1}+g^{r} \pi^{r-1} \mu^{r-1} \\
& =d_{Z}^{r} g^{r+1}+g^{\prime} \mu^{r-1} \\
& =w^{r}
\end{aligned}
$$

completes the induction.

1.5. The following corollary is an immediate consequence of the theorem. 
Corollary. Let $A$ be a finite-dimensional k-algebra. Then $D^{b}(A)$ has AuslanderReiten triangles if and only if $A$ has finite global dimension.

\section{RePetitive ALGebras}

For the reader's convenience we recall relevant definitions, but refer to $[\mathrm{H} 1$, $\mathrm{H} 2$ ] for more detail and proofs of some elementary facts.

2.1. Let $A$ be a finite-dimensional $k$-algebra, where $k$ is some field. Denote by $D=\operatorname{Hom}_{k}(-, k)$ the standard duality on $\bmod A$ the category of finitely generated left $A$-modules. Let $Q=D A$. Then $Q$ is an injective cogenerator and an $A$-bimodule: given $a, b \in A$ and $\varphi \in Q$ then $a \varphi b$ is the $k$-linear map which sends $\lambda \in A$ to $\varphi(b \lambda a)$.

Let us construct the repetitive algebra $\hat{A}$. It will be a selfinjective algebra and always infinite-dimensional (except in the trivial case $A=0$, which we exclude).

The underlying vectorspace of $\hat{A}$ is given by

$$
\hat{A}=\left(\bigoplus_{i \in \mathbb{Z}} A\right) \oplus\left(\bigoplus_{i \in \mathbb{Z}} Q\right)
$$

We denote the elements of $\hat{A}$ by $\left(a_{i}, \varphi_{i}\right)_{i}$, where $a_{i} \in A, \varphi_{i} \in Q$, of course with almost all $a_{i}, \varphi_{i}$ being zero. The multiplication is defined by

$$
\left(a_{i}, \varphi_{i}\right)_{i} \cdot\left(b_{i}, \psi_{i}\right)_{i}=\left(a_{i} b_{i}, a_{i+1} \psi_{i}+\varphi_{i} b_{i}\right)_{i} .
$$

We refer to [H1] for an interpretation of $\hat{A}$ as doubly infinite matrix algebra.

We define an $\hat{A}$-module $X$ as a sequence $X=\left(X_{n}, f_{n}\right)$ of $A$-modules $X_{n}$ and $A$-linear maps $f_{n}: X_{n} \rightarrow \operatorname{Hom}_{A}\left(Q, X_{n+1}\right)$ satisfying $f_{n-1} \cdot \operatorname{Hom}_{A}\left(Q, f_{n}\right)=$ 0 for all $n \in \mathbb{Z}$. Instead of $\left(X_{n}, f_{n}\right)$ we also write

$$
\cdots X_{-2} \stackrel{f_{-2}}{\curvearrowright} X_{-1} \stackrel{f_{-1}}{\curvearrowright} X_{0} \curvearrowright \stackrel{f_{0}}{\curvearrowright} X_{1} \stackrel{f_{1}}{\curvearrowright} X_{2} \cdots
$$

A morphism $h: X=\left(X_{n}, f_{n}\right) \rightarrow Y=\left(Y_{n}, g_{n}\right)$ is a sequence $h=\left(h_{n}\right)$ of $A$-linear maps $h_{n}: X_{n} \rightarrow Y_{n}$ such that the following diagrams commute for all $n \in \mathbb{Z}$.

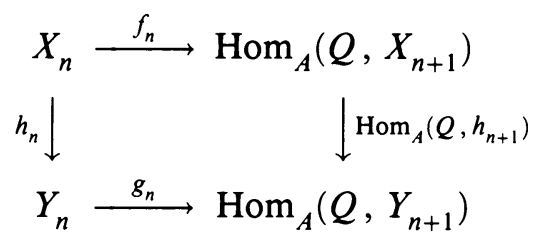

We denote by $\bmod \hat{A}$ the category of all $\hat{A}$-modules $X=\left(X_{n}, f_{n}\right)$ such that $\operatorname{dim}_{k}\left(\bigoplus_{n} X_{n}\right)<\infty$.

It is quite easy to see that $\bmod \hat{A}$ is a Frobenius category in the sense of [H1]. In fact, the indecomposable projective-injective $\hat{A}$-modules are given by

$$
\cdots 0 \curvearrowright X_{i} \stackrel{f_{i}}{\curvearrowright} X_{i+1} \curvearrowright 0 \cdots
$$


where $X_{i+1}$ is an indecomposable $A$-injective module, $X_{i}=\operatorname{Hom}_{A}\left(Q, X_{i+1}\right)$, and $f_{i}=i d_{X_{i}}$. By $\underline{\bmod } \hat{A}$ we denote the associated stable category. Thus the objects in $\bmod \hat{A}$ coincide with the objects in $\bmod \hat{A}$, while the morphisms are given by $\underline{\operatorname{Hom}}(X, Y)=\operatorname{Hom}(X, Y) / I(X, Y)$ where for $\hat{A}$-modules $X, Y$ we have denoted by $I(X, Y)$ the subspace of those morphisms which factor over an injective $\hat{A}$-module.

It was shown in $[\mathrm{H} 1]$ that $\bmod \hat{A}$ is a triangulated category, where the suspension functor serves as the translation functor. We will denote its application on a module $X \in \underline{\bmod } \hat{A}$ by $X[1]$.

2.2. Let us now relate this description to the one given in the introduction.

The trivial extension algebra $T(A)$ of $A$ by $Q$ is the following finitedimensional $k$-algebra. The additive structure is $A \oplus Q$ and the multiplication is defined by

$$
(a, \varphi) \cdot(b, \psi)=(a b, a \psi+\varphi b)
$$

for $a, b \in A$ and $\varphi, \psi \in Q$.

The algebra $T(A)$ is a $\mathbb{Z}$-graded algebra, where the elements of $A \oplus 0$ are the elements of degree 0 and those of $0 \oplus Q$ the elements of degree 1 . We denote by $\bmod ^{\mathbb{Z}} T(A)$ the category of finitely generated $\mathbb{Z}$-graded $T(A)$-modules with morphisms of degree zero. The following is a straightforward observation.

The categories $\bmod \hat{A}$ and $\bmod ^{\mathbb{Z}} T(A)$ are equivalent.

2.3. Now we are able to prove the theorem mentioned in the introduction.

Theorem. Let $A$ be a finite-dimensional k-algebra. $D^{b}(A)$ is triangle-equivalent to $\underline{\bmod } \hat{A}$ if and only if gl.dim $A<\infty$.

Proof. If gl.dim $A<\infty$ a proof was given in [H1].

For the converse suppose that $G: D^{b}(A) \rightarrow \underline{\bmod } \hat{A}$ is a triangle-equivalence. By [AR] we infer that $\underline{\bmod } \hat{A}$ has Auslander-Reiten triangles. Thus $D^{b}(A)$ has Auslander-Reiten triangles. So the assertion now follows from 1.5.

2.4. We have a canonical embedding $\phi$ of $\bmod A$ into $\bmod \hat{A}$ which sends a module $X \in \bmod A$ onto $\left(X_{n}, f_{n}\right)$ where $X_{0}=X$ and $X_{n}=0$ for $n \neq 0$. We infer that $\phi$ is exact.

The following two facts were established in [H2].

The composition of $\phi$ with the canonical functor $\bmod \hat{A} \rightarrow \underline{\bmod } \hat{A}$ is a full embedding.

Moreover we have for $X, Y \in \bmod A$ and $i \in \mathbb{Z}$ that

$$
\underline{\operatorname{Hom}}(\phi(X),(\phi(Y))[i]) \simeq \operatorname{Ext}_{A}^{i}(X, Y)
$$

2.5. In [H1], (see also [H2]) we have constructed a full and faithful exact functor $F$ of triangulated categories $F: D^{b}(A) \rightarrow \underline{\bmod } \hat{A}$ such that $F$ extends the identity functor on $\bmod A$. A considerable simplification was obtained in $[\mathrm{KV}]$. We will now give a very easy construction. 
Theorem. Let $A$ be a finite-dimensional k-algebra. Then there exists a full and faithful exact functor $F$ of triangulated categories $F: D^{b}(A) \rightarrow \bmod \hat{A}$ such that $F$ extends the identity functor on $\bmod A$.

Proof. Let $\phi: \bmod A \rightarrow \bmod \hat{A}$ be the exact functor defined in 2.4. Then $\phi$ extends to an exact functor $\tilde{\phi}: D^{b}(A) \rightarrow D^{b}(\hat{A})$. Now consider the localisation sequence as constructed in [Ri2]:

$$
K^{b}\left({ }_{\hat{A}} \mathscr{P}\right) \stackrel{\mu}{\longrightarrow} D^{b}(\hat{A}) \stackrel{\pi}{\longrightarrow} \underline{\bmod } \hat{A} .
$$

Set $F=\pi \tilde{\phi}$. Then $F$ is an exact functor, which extends the identity functor on $\bmod A$. It follows from 2.4. that $F$ is full and faithful by using the Beilinson lemma [B].

\section{ACKNOWLEDGMENT}

These results were obtained while the author was visiting the University of Trondheim. And I would like to thank I. Reiten for the invitation and both she and $M$. Auslander for the discussions which provoked these investigations.

\section{REFERENCES}

[AR] M. Auslander and I. Reiten, Stable equivalence of dualizing $R$-varieties, Adv. in Math. 12 (1974), 306-366.

[B] A. Beilinson, Coherent sheaves on $\mathbb{P}^{n}$ and problems of linear algebra, Funct. Anal. Appl. 12 (1979), 214-216.

[H1] D. Happel, On the derived category of a finite-dimensional algebra, Comment. Math. Helv. 62 (1987), 339-389.

[H2] _ Triangulated categories in the representation theory of finite-dimensional algebras, Cambridge University Press 119 (1988).

[KV] B. Keller and D. Vossieck, Sous les catégories dérivées, C.R. Acad. Sci. Paris t. 305, Série I (1987), 225-228.

[R] C. M. Ringel, Tame algebras and integral quadratic forms, Lecture Notes in Math., vol. 1099, Springer, 1984.

[Ri1] J. Rickard, Morita theory for derived categories, J. London Math. Soc 39 (1989), 436-456.

[Ri2] _ Derived categories and stable equivalence, J. Pure Appl. Algebra 61 (1989), 303-317.

[TW] H. Tachikawa and T. Wakamatsu, Cartan matrices and Grothendieck groups of stable categories, preprint.

[V] J. L. Verdier, Catégories dérivées, état 0, Lecture Notes in Math., vol. 569, Springer, 1977, pp. 262-311.

FAKUltät Für MAThematik, Universität Bielefeld, D 4800 Bielefeld 1, UniversitätsSTR.25, Federal RePUblic OF GeRmany 